\title{
Characterization of bacteriophages infecting clinical isolates of Pseudomonas aeruginosa stored in a culture collection
}

\author{
C.C.S. Zanetti ${ }^{1}$, R.C.C. Mingrone ${ }^{1}$, J.J. Kisielius ${ }^{2}$, M. Ueda-Ito ${ }^{2}$ and A.C.C. Pignatari ${ }^{1}$ \\ ${ }^{1}$ Laboratório Especial de Microbiologia Clínica, Divisão de Doenças Infecciosas, Universidade Federal de São Paulo, \\ São Paulo, SP, Brasil \\ ${ }^{2}$ Núcleo de Microscopia Eletrônica, Instituto Adolfo Lutz, São Paulo, SP, Brasil
}

\begin{abstract}
Some clinical isolates of Pseudomonas aeruginosa stored in our culture collection did not grow or grew poorly and showed lysis on the culture plates when removed from the collection and inoculated on MacConkey agar. One hypothesis was that bacteriophages had infected and killed those clinical isolates. To check the best storage conditions to maintain viable $P$. aeruginosa for a longer time, clinical isolates were stored at various temperatures and were grown monthly. We investigated the presence of phage in 10 clinical isolates of $P$. aeruginosa stored in our culture collection. Four strains of $P$. aeruginosa were infected by phages that were characterized by electron microscopy and isolated to assess their ability to infect. The best condition to maintain the viability of the strains during storage was in water at room temperature. Three Siphoviridae and two Myoviridae phages were visualized and characterized by morphology. We confirmed the presence of bacteriophages infecting clinical isolates, and their ability to infect and lyse alternative hosts. Strain PAO1, however, did not show lysis to any phage. Mucoid and multidrug resistant strains of $P$. aeruginosa showed lysis to $50 \%$ of the phages tested.
\end{abstract}

Key words: Bacteriophages; Pseudomonas aeruginosa

\section{Introduction}

Bacteriophages or "phages" are viruses that specifically infect bacteria and replicate within them (1). Phages do not have their own metabolism, therefore they need material resources and host metabolism and energy to replicate (2). There is no universal method of virus classification. The current phage classification, developed by the International Committee on Taxonomy of Viruses (ICTV) is based on a set of properties that may or may not be present in a particular member. This classification includes one order, 14 families and 37 genera (3). The genetic diversity of phages is extremely high. The similarity of bacteriophage nucleotide sequences isolated from different bacterial hosts or even from common hosts is small or not identifiable $(3,4)$.

Pseudomonas bacteriophages have been studied for decades. They have been used in phage typing and therapy (5). They appear pleomorphic and tailed by electron microscopy. The order Caudovirales includes three families of phages, Myoviridae, Siphoviridae and Podoviridae that infect bacteria of the genus Pseudomonas. Most phages observed by electron microscopy belong to the family Siphoviridae (6), which includes phages B3 and D3112, commonly isolated from natural populations and clinical isolates of Pseudomonas aeruginosa $(7,8)$.

The Laboratório Especial de Microbiologia Clínica is a reference laboratory in clinical microbiology and stores bacteria isolated from patients from different regions of Brazil and other countries. Some strains of $P$. aeruginosa, when removed from the culture collection and inoculated onto MacConkey agar to be used in research projects or to be sent to other laboratories, did not grow or grew poorly, sometimes showing signs of lysis on the plate. One hypothesis was that the clinical isolates of $P$. aeruginosa stored in the laboratory's culture collection were not viable due to the presence of bacteriophages infecting and killing these samples.

In order to investigate this hypothesis we checked the best storage conditions to maintain viable $P$. aeruginosa for a longer time and tried to demonstrate the presence of bacteriophages infecting the stored clinical isolates of

Correspondence: C.C.S. Zanetti, Laboratório Especial de Microbiologia Clínica, Rua Leandro Dupret, 188, 04025-010 São Paulo, SP, Brasil. Fax: +55-11-5571-5180. E-mail: cyntheacarolina@ig.com.br 
$P$. aeruginosa. We looked for bacteriophages in $P$. aeruginosa by electron microscopy and observed the ability of bacteriophages to infect antibiotic-sensitive and -resistant $P$. aeruginosa strains.

\section{Material and Methods}

\section{Phage and bacterial strains}

Ten clinical isolates of $P$. aeruginosa that showed plaques on MacConkey agar (presumably infected by lysogenic bacteriophages), when they were streak seeded and incubated at $37^{\circ} \mathrm{C}$ overnight after being received at the laboratory, were included in this study. These bacteria were selected from isolates obtained between November 2008 and March 2009 (Table 1). Phages included in this study were extracted from these clinical isolates. We included three clinical isolates of $P$. aeruginosa that did not show lysis on the inoculation plate as alternative hosts. All clinical strains were isolated from Brazilian hospital patients in various years (Table 1). PAO1 was also included as an alternative host.

Lambda phage::Tn5 and its propagating strain Escherichia coli E7026 were used as a positive control and were kindly provided by Dr. Rosa Maria Silva, Departamento de Microbiologia e Imunologia Básica, Universidade Federal de São Paulo, São Paulo, SP, Brazil.

Influence of different storage conditions on the maintenance of strains and on plaque formation

About five colonies of each clinical isolate of $P$. aeruginosa, including the strains suspected to be infected with phages and the three clinical isolates used as alternative hosts, were grown on MacConkey agar (Oxoid, UK) at $37^{\circ} \mathrm{C}$ overnight. All strains had been previously isolated at a hospital or laboratory as shown in Table 1. Each clinical isolate was inoculated into four sterile tubes containing $1.0 \mathrm{~mL}$ sterile deionized water and into three sterile tubes containing $1.0 \mathrm{~mL}$ sterile Tryptic Soy Broth (TSB, Oxoid) with 15\% glycerol. Each tube was inoculated with a $10-\mu \mathrm{L}$ loop fresh culture. The four tubes containing the isolate and deionized water were stored at room temperature, $4^{\circ},-20^{\circ}$ or $-70^{\circ} \mathrm{C}$. The three tubes containing the isolate and sterile TSB with $15 \%$ glycerol were stored at $4^{\circ},-20^{\circ}$ or $-70^{\circ} \mathrm{C}$. The isolates were seeded monthly for 12 months on MacConkey agar using a $10-\mu \mathrm{L}$ disposable loop, and incubated at $37^{\circ} \mathrm{C}$ for $24 \mathrm{~h}$. Plates were checked monthly for bacterial growth and the occurrence of spontaneous lysis. The presence of a single colony was considered bacterial growth.

We calculated the number of isolates that were not viable (i.e., "dead") at the end of the study. The recovery percentage of the isolates (i.e., number of isolates that grew when they were removed from storage and seeded) was calculated each month during the 12 months of the study. We considered bacteria dead if they did not grow on the culture medium for three or more consecutive samplings until the end of the 12 months of evaluation. If there were three consecutive samplings without growth, but growth resumed later, we considered the bacteria viable.

\section{Obtaining plaques}

Lysogenic phages were extracted from the 10 clinical isolates as previously described. This protocol was developed independently of the evaluation of the influence

Table 1. Clinical isolates of Pseudomonas aeruginosa included in this study.

\begin{tabular}{lcccc}
\hline Collection No. & Origin & Site & Storage data & Note \\
\hline P1088 & Graac & Blood culture & $03 / 01 / 2001$ & Alternative host \\
P7960 & Outpatient & Urine & $03 / 15 / 2007$ & Alternative host \\
P9582 & HSP & Blood culture & $11 / 11 / 2008$ & \\
P9583 & HSP & Blood culture & $11 / 11 / 2008$ & Alternative host \\
P9611 & HSP & Catheter tip & $11 / 19 / 2008$ & \\
P9758 & HSP & Peritoneal fluid & $01 / 27 / 2009$ & \\
P9715 & HSP & Tracheal aspirate & $01 / 06 / 2009$ & $12 / 17 / 2008$ \\
P9705 & HBB & Blood culture & $02 / 27 / 2009$ & \\
P9808 & HSP & Tracheal aspirate & $02 / 27 / 2009$ & \\
P9809* & HSP & Blood culture & $02 / 27 / 2009$ & \\
P9810 & HSP & Urine & $02 / 27 / 2009$ & \\
P9812* & HSP & Tracheal aspirate & $03 / 03 / 2009$ & \\
P9842 & HRH & Urine & \\
\hline
\end{tabular}

Graac: Grupo de Apoio ao Adolescente e à Criança com Câncer; HSP: Hospital São Paulo; HBB: Hospital Base de Brasília; HRH: Hospital do Rim e Hipertensão. The clinical isolate P1088 is resistant to imipenem and produces the metallo- $\beta$-lactamase SPM-1; P7960 is a mucoid strain, resistant to carbapenem, but susceptible to ceftazidime; P9758 is a clinical isolate susceptible to all betalactamic antibiotics; P9809* and P9812* were collected from the same patient but from different body sites. 
of different storage conditions on the maintenance of strains and formation of plaques. The overlay method protocol used has already been described (9), but was used with some modifications.

The 10 clinical isolates and the E. coli E7026 propagating strain were grown in Luria Bertani broth medium (LB, Sigma, USA) with shaking at $110 \mathrm{rpm}$ and $37^{\circ} \mathrm{C}$ until reaching an absorbance $\left(A_{640}\right)$ of 1.0 . Then, $1.0 \mathrm{~mL}$ of each $P$. aeruginosa culture was incubated at $37^{\circ} \mathrm{C}$ for $15 \mathrm{~min}$. $A$ $0.5-\mathrm{mL}$ sample of the $\mathrm{E} 7026$ suspension was mixed with $0.5 \mathrm{~mL}$ lambda phage and incubated at $37^{\circ} \mathrm{C}$ for $15 \mathrm{~min}$. The clinical strains were not infected with the lambda phage because it was assumed that they were already infected by lysogenic phages. A $0.2-\mathrm{mL}$ sample of each suspension was mixed with $3.0 \mathrm{~mL}$ melted top agar cooled to $45^{\circ} \mathrm{C}$ in sterile glass tubes.

The mixtures were prepared in duplicate. Each mixture was poured onto a Petri dish containing solid LB with $1.5 \%$ agarose, and all plates were incubated at $37^{\circ} \mathrm{C}$ for $18-24 \mathrm{~h}$. Bacterial growth and the presence of plaques were observed.

\section{Preparation of phage stock suspensions}

The protocol used has already been described (10), but was used with some modifications. We added $3.0 \mathrm{~mL}$ phage buffer (1 M Tris- $\mathrm{HCl}, \mathrm{pH} 7.5,10 \mathrm{mM} \mathrm{MgSO}_{4}$, $10 \mathrm{mM} \mathrm{NaCl}, 2.0 \%$ gelatin) to each plate with plaques (obtained as described) and incubated them at $4{ }^{\circ} \mathrm{C}$ for $24 \mathrm{~h}$. The liquid phase was collected and centrifuged for $30 \mathrm{~min}$ at $4500 \mathrm{~g}$ and $4^{\circ} \mathrm{C}$. The supernatant was filtered $\left(0.22-\mu \mathrm{L}\right.$ membranes, Millipore ${ }^{\mathbb{R}}$, USA) and the phage stock suspensions were stored at $4^{\circ} \mathrm{C}$. Duplicates from cultures of the same bacterium were collected in the same tube for processing.

\section{Infection of alternative hosts by phages}

The alternative hosts were infected with phage stock suspensions obtained as described above. The ability of each phage to infect the alternative hosts and cause lysis was tested. E. coli E7026 and lambda phage::Tn5 were used as a positive control test for lysis. Alternative hosts (E7026, PAO1, P1088, P7960, and P9758) were seeded on MacConkey agar and incubated at $37^{\circ} \mathrm{C}$ overnight. Three to five colonies of each bacterium were inoculated into $3.0 \mathrm{~mL}$ TSB and incubated at $37^{\circ} \mathrm{C}$ overnight. Bacterial suspensions were then diluted $1: 10$ with TSB, and $0.2 \mathrm{~mL}$ of each dilution was seeded on MacConkey agar using a Drigalsky handle to obtain confluent growth. On these plates, $0.02 \mathrm{~mL}$ of each phage stock suspension was spotted with a pipette and disposable tips. After drying at room temperature, the plates were incubated at $37^{\circ} \mathrm{C}$ for 16-18 $\mathrm{h}$ and then observed for the presence of plaques.

\section{Electron microscopy}

Bacteriophage particles were negatively stained, examined by electron microscopy at Instituto Adolfo Lutz and characterized by morphology as outlined by Ackermann (3). The specimen grids were prepared from plaques obtained by the method described above. Briefly, viral particles were adhered to 300-mesh formvar/carboncoated copper grids. The electron micrographs were obtained with a JEOL JEM-1011 transmission electron microscope operating at $80 \mathrm{kV}$, and the electron micrographs were recorded with a charge-coupled device camera (model 785 ES1000W, Gatan, USA) and the Gatan version 1.6 program. About five measurements were made for each phage examined.

The protocol used to prepare the grids has been described $(11,12)$, but some modifications were made. Grids were placed for 10 min on a drop of $1 \%$ Alcian blue to make them more hydrophilic, dried with filter paper and washed three times with distilled water. The grid remained in distilled water until used. Fifty microliters of $2 \%$ potassium phosphotungstate (PTK), pH 6.4, was added directly onto a plate. The PTK was mixed by scraping the plaques on the plates gently with the pipette tip. The grid was removed from the water; the excess water was removed and the grid was placed in contact with the resuspended plaques in the PTK. The grid was then washed in a drop of PTK and dried with filter paper, and remained at least $24 \mathrm{~h}$ at room temperature before the analysis by electron microscopy.

\section{Results}

\section{Influence of different storage conditions on maintenance of strains and plaque formation}

The best storage condition for the $P$. aeruginosa clinical isolates was in water at room temperature (Table 2). All isolates were viable at the end of the study and only one isolate $(0.6 \%)$ did not grow in 1 of the 12 months of evaluation. We considered dead bacteria to be those that did not grow for three or more consecutive subcultures until the end of the 12 months of evaluation. There were 13 isolates stored at each storage condition that were subcultured 12 times each. Altogether, there were 156 subcultures from each storage condition, but one that was stored in water at room temperature did not grow, therefore, as mentioned above, 155 of 156 subcultures (99.4\%) grew bacteria. The largest number of samples with formation of plaques (80/156 subcultures) was from bacteria stored in water at $4^{\circ} \mathrm{C}$ or TSB with glycerol at $4^{\circ} \mathrm{C}$. TSB with glycerol at $-70^{\circ} \mathrm{C}$ was the storage condition with the lowest number of samples with plaque formation (12/156).

\section{Infection of alternative hosts by the phages}

The ability of the extracted bacteriophages to infect the alternative bacterial strains was restricted mainly to the P7960 mucoid, multidrug-resistant $P$. aeruginosa strain, which was lysed by $50 \%$ of the phages tested (Table 3). PAO1 was not lysed by any of the phages. 
Table 2. Evaluation of the influence of different storage conditions on the maintenance of Pseudomonas aeruginosa isolates.

\begin{tabular}{lccc}
\hline Storage condition & $\begin{array}{c}\text { Isolates not viable at the } \\
\text { end of the study }(\mathrm{n})\end{array}$ & $\begin{array}{c}\text { Isolates recovered } \\
\text { during the study (\%) }\end{array}$ & $\begin{array}{c}\text { Growth with formation } \\
\text { of plaques }(\mathrm{n})\end{array}$ \\
\hline Water at RT & 0 & 99.4 & 60 \\
Water at $4{ }^{\circ} \mathrm{C}$ & 1 & 98.1 & 80 \\
Water at $-20^{\circ} \mathrm{C}$ & 10 & 37.2 & 27 \\
Water at $-70^{\circ} \mathrm{C}$ & 4 & 82.7 & 66 \\
$\mathrm{TSB}+$ glycerol at $4^{\circ} \mathrm{C}$ & 1 & 96.8 & 80 \\
$\mathrm{TSB}+$ glycerol at $-20^{\circ} \mathrm{C}$ & 3 & 78.8 & 42 \\
TSB + glycerol at $-70^{\circ} \mathrm{C}$ & 8 & 55.8 & 12 \\
\hline
\end{tabular}

RT: room temperature; TSB: Tryptic Soy Broth.

\section{Electron microscopy}

Bacteriophages were seen in 5 of $10 P$. aeruginosa clinical isolates that we suspected to be infected (P9583, P9705, P9808, P9810, and P9842). Family Siphoviridae bacteriophages (with flexible tails) were found on grids obtained from clinical isolates P9583, P9808, and P9842 (Figure 1), and family Myoviridae bacteriophages (with a neck and contractile tail) were found on grids obtained from clinical isolates P9705 and P9810 (Figure 2). Probable capsids or tails were visualized in the other 4 $P$. aeruginosa clinical isolates: P9582, P9809, P9812, and P9715 (data not shown). Bacteriophages were not observed on grids obtained from sample P9611.

\section{Discussion}

We suspect that phages influenced the survival of the bacteria. Host nutritional and metabolic status are critical for viral infection and proliferation, affecting adsorption, replication, lytic activity, and phage survival. Several studies have shown that the highest rates of phage proliferation and production were observed under conditions ideal for host growth. The low availability of nutrients resulted in an increased latency period and reduced

Table 3. Analysis of alternative host infection.

\begin{tabular}{lcccc}
\hline Phage & P1088 & P7960 & P9758 & PAO1 \\
\hline 9582 & - & - & + & - \\
9583 & - & + & - & - \\
9611 & - & - & - & - \\
9705 & - & + & - & - \\
9715 & + & - & - & - \\
9808 & + & + & + & - \\
9809 & - & + & + & - \\
9810 & - & - & - & - \\
9812 & - & - & + & - \\
9842 & - & + & + & - \\
\hline
\end{tabular}

-: no lysis; +: lysis. release of phage particles (13-16). Phage particles can also be formed during the host stationary phase; however, cell lysis can be interrupted (2).

Starvation for as long as 5 years does not prevent viral reproduction in all host species, although cell lysis is delayed and burst size reduced (2). We suspect that, during storage in water, the low availability of nutrients results in an increased phage latency period, reduced release of phage particles, or formation of phage particles with interruption of lysis. Therefore, when bacteria are grown in a rich medium, the lytic pathway is favored and cell lysis occurs.

Due to spontaneous lysis in the initial monthly subcultures during evaluation of bacterial storage conditions, initial phage stock suspensions were obtained without need to induce the lytic cycle with UV light or mitomycin. We rationalized that the temperate phages present in $P$. aeruginosa clinical isolates entered in a lytic cycle when seeded after extended starvation. In the course of time, lysis became less frequent perhaps because of the successive thawings and seedings, and we tried to induce the lytic cycle by heating the bacterial culture to $45^{\circ} \mathrm{C}$

According to Sambrook and Russell (17), $\lambda$ phage carrying a mutation in the $\mathrm{cl}$ gene can be induced to enter the lytic cycle by heating the bacterial culture at $45^{\circ} \mathrm{C}$, since heating at this temperature partially inactivates this gene. Under normal conditions, the $\mathrm{cl}$ gene inhibits expression of lytic functions. We suppose that this also occurs with $P$. aeruginosa phage, but at a higher temperature $\left(50^{\circ} \mathrm{C}\right)$, because $\lambda$ phage is a coliphage and we are investigating Pseudomonas phage.

Initially, it was difficult to define the methodology to be used to assess the best storage condition. We tried to reproduce conditions in the study protocol that were exactly the same as those in our culture collection. The best condition found to store the strains, water at room temperature, was already being used in our laboratory. It is important to maintain the viability of strains, especially during storage, because they are used in national and international surveillance, research projects in bacterial 


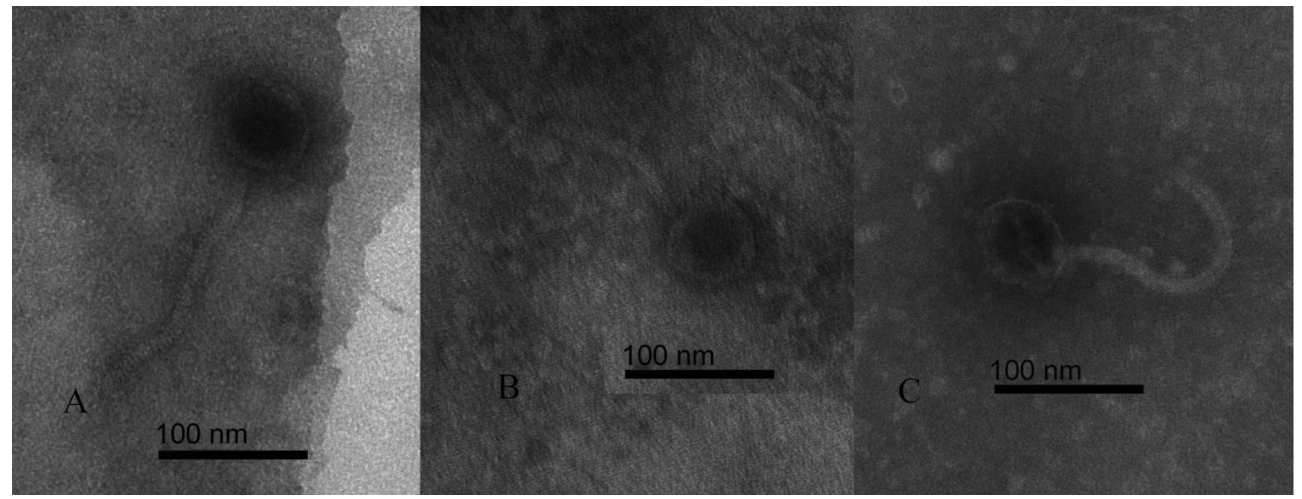

Figure 1. Family Siphoviridae bacteriophages isolated from clinical samples. The scale bar corresponds to $100 \mathrm{~nm}$. $A$, Phage isolated from Pseudomonas aeruginosa P9583. B, Phage isolated from P. aeruginosa P9808. C, Phage isolated from P. aeruginosa P9842.

resistance to antimicrobials, and investigations of mechanisms of resistance.

The plaques seen on MacConkey agar that aroused interest in the investigation of phages could also be due to autolysis of $P$. aeruginosa, which can be an adaptive behavior mediated by an extracellular signal of the Quorum Sensing hierarchy. There is a correlation between strains with pronounced lysis and overproduction of the Pseudomonas quinolone signal, an extracellular signal that interacts with Quorum Sensing, and the mechanism of autolysis may involve activation of endogenous prophage (18).

The alternative host and control strain, PAO1, widely used as a propagator strain in studies involving $P$. aeruginosa bacteriophages, did not show lysis by any phage released by our clinical isolates. However, we cannot state that this strain was resistant to phages. Perhaps some phage had infected PAO1 but entered a lysogenic pathway, or actually no phage infected this bacterium. There may be more phages in nature than thought. Some studies have used PAO1 primarily to

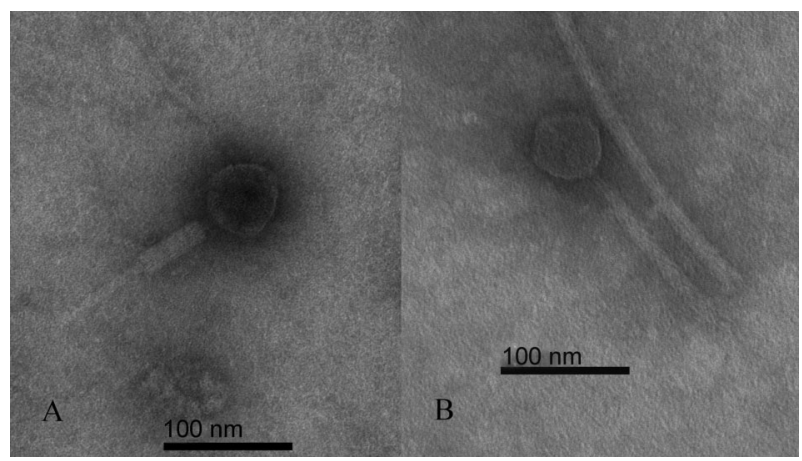

Figure 2. Family Myoviridae bacteriophages isolated from clinical samples. The scale bar corresponds to $100 \mathrm{~nm}$. A, Phage isolated from Pseudomonas aeruginosa P9705. B, Phage isolated from $P$. aeruginosa P9810. select lytic phages from water or the environment (19-21) but there are many phages in the environment that do not infect PAO1 or infect it but did not show lysis (i.e., lysogenic phages).

The extracted phages used in this study are temperate, and may have integrated their genomes into the PAO1 chromosome to establish a prophage state without undergoing a lytic interaction. We suggest that further studies using the agar overlay method with PAO1 and extracted phages be performed with top agar melted and cooled to $50^{\circ} \mathrm{C}$ during the inoculation of phage in the bacteria. A suitable protocol has been described recently (5).

The phage extracted from $P$. aeruginosa P9808 infected and caused lysis in 3 of the 4 strains tested as alternative hosts, suggesting that this phage has a common gateway in these strains and that essential components of the cell surface may facilitate the phage adhesion. The isolate P7960, a mucoid strain, showed lysis by $50 \%$ of the phages tested. Mucoid strains in patients with cystic fibrosis are difficult to eradicate. The alginate production and biofilm formation by these strains contribute significantly to the resistance to treatment regimens and host defenses, resulting in a poor prognosis for patients with cystic fibrosis (22). Our hypothesis about this finding is that some changes in the bacterial cell wall and membrane may make it more permissive to the interaction between the capsid and the membrane protein, since the molecules recognized by phage usually are essential components of cell surface $(2,23)$. The adhesion of phage to a host cell can employ structural material (glycoproteins and lipopolysaccharides), transport machinery (porins, amino acids or complex sugar transporters), and interaction apparatus between cells (F pili) depending on the phage type. Receptors responsible for adsorption in situ remain unknown $(2,24)$.

The evidence that the mucoid strain showed lysis to half of the phages tested reinforces the idea that phage therapy can be an effective alternative for the treatment or prevention of infections caused by multiresistant bacteria 
(25). Mucoid strains of $P$. aeruginosa are difficult to be eradicated with treatment, mainly in patients with cystic fibrosis $(22,26)$. This finding has aroused our interest and we intend to begin study of such samples. Our hypothesis regarding the mucoid strain susceptibility to phage infection is that some changes in the cell wall and membrane of the bacteria may make it more permissive to the interaction between the capsid and membrane proteins, again because the molecules recognized by phages are usually essential components of the cell surface $(2,23)$.

Phage particles were only seen by electron microscopy when we used the protocol developed at Instituto Adolfo Lutz, in which the grids were prepared directly from plaques. We tried to obtain micrographs directly from the phage stock suspensions, but were not successful. We assume that this occurred due to the low titer of phage particles, despite the stock suspension having been obtained from numerous lysis plates after inoculation of the bacterial culture on top agar melted and cooled to $45^{\circ} \mathrm{C}$. No phage particles were visualized infecting sample P9611, but it is not possible to state that this bacterium was not infected because the phage DNA might have been present in the prophage state.

The phages were classified only based on morphology.

\section{References}

1. Fischetti VA. Bacteriophage lysins as effective antibacterials. Curr Opin Microbiol 2008; 11: 393-400, doi: 10.1016/j.mib. 2008.09.012.

2. Weinbauer MG. Ecology of prokaryotic viruses. FEMS Microbiol Rev 2004; 28: 127-181, doi: 10.1016/j.femsre. 2003.08.001.

3. Ackermann HW. Phage classification and characterization. Methods Mol Biol 2009; 501: 127-140, doi: 10.1007/978-160327-164-6_13.

4. Comeau AM, Hatfull GF, Krisch HM, Lindell D, Mann NH, Prangishvili D. Exploring the prokaryotic virosphere. Res Microbiol 2008; 159: 306-313, doi: 10.1016/j.resmic.2008. 05.001.

5. Ceyssens PJ. Isolation and characterization of lytic bacteriophages infecting Pseudomonas aeruginosa. [PhD's thesis]. Leuven: Katholieke Universiteit Leuven; 2009.

6. Ackermann HW. 5500 Phages examined in the electron microscope. Arch Virol 2007; 152: 227-243, doi: 10.1007/ s00705-006-0849-1.

7. Heo YJ, Chung IY, Choi KB, Lau GW, Cho YH. Genome sequence comparison and superinfection between two related Pseudomonas aeruginosa phages, D3112 and MP22. Microbiology 2007; 153: 2885-2895, doi: 10.1099/ mic.0.2007/007260-0.

8. Zegans ME, Wagner JC, Cady KC, Murphy DM, Hammond $\mathrm{JH}$, O'Toole GA. Interaction between bacteriophage DMS3 and host CRISPR region inhibits group behaviors of Pseudomonas aeruginosa. J Bacteriol 2009; 191: 210-219, doi: 10.1128/JB.00797-08.

9. Sillankorva SM. Utilização de bacteriófagos no controle de células suspensas e biofilmes de Pseudomonas fluorescens.
Because the ICTV's virus taxonomy has been continually under review, we classified our phages only by order and family according to Ackermann (3). Three different phage morphotypes were visualized, suggesting that the bacterial strains already had prophages when isolated from patients and that there was not a common source of phage contamination during the storage or manipulation of the clinical isolates. It would be interesting to investigate whether the phages found with the same morphotype show the same DNA restriction profile or the same gene sequence.

Lysogenic phages extracted from bacterial samples are not suitable for phage therapy studies because they have the property of integrating themselves into the bacteria. Pseudomonas lytic phages are the most suitable $(27,28)$, and can be obtained from environmental samples of water, including sewage, ocean and fresh water, and soil $(5,6)$.

\section{Acknowledgments}

We thank the staff of Laboratório Central, Hospital São Paulo, Universidade Federal de São Paulo, and the staff of Centro de Microscopia Eletrônica (CEME), Universidade Federal de São Paulo.

[Master's thesis]. Braga: University of Minho; 2004.

10. Bergan T. Methods in microbiology. Vol. 10. Norris JR (editor), New York: Academic Press; 1978.

11. Brenner S, Horne RW. A negative staining method for high resolution electron microscopy of viruses. Biochim Biophys Acta 1959; 34: 103-110, doi: 10.1016/0006-3002(59)90237-9.

12. Burbano-Rosero EM, Ueda-Ito M, Kisielius JJ, NagasseSugahara TK, Almeida BC, Souza CP, et al. Diversity of somatic coliphages in coastal regions with different levels of anthropogenic activity in Sao Paulo State, Brazil. Appl Environ Microbiol 2011; 77: 4208-4216, doi: 10.1128/ AEM.02780-10.

13. Kokjohn TA, Sayler GS, Miller RV. Attachment and replication of Pseudomonas aeruginosa bacteriophages under conditions simulating aquatic environments. J Gen Microbiol 1991; 137: 661-666, doi: 10.1099/00221287-1373-661.

14. Lenski RE. Dynamics of interactions between bacteria and virulent bacteriophage. Adv Microb Ecol 1988; 10: 1-44, doi: 10.1007/978-1-4684-5409-3_1.

15. Middelboe M. Bacterial growth rate and marine virus-host dynamics. Microb Ecol 2000; 40: 114-124.

16. Proctor LM, Okubo A, Fuhrman JA. Calibrating estimates of phage-induced mortality in marine bacteria: ultrastructural studies of marine bacteriophage development from onestep growth experiments. Microb Ecol 1993; 25: 161-182, doi: $10.1007 / B F 00177193$.

17. Sambrook J, Russell D. Molecular cloning: a laboratory manual. 3rd edn. New York: CSHL Press; 2001.

18. D'Argenio DA, Calfee MW, Rainey PB, Pesci EC. Autolysis and autoaggregation in Pseudomonas aeruginosa colony 
morphology mutants. J Bacteriol 2002; 184: 6481-6489, doi: 10.1128/JB.184.23.6481-6489.2002.

19. Ceyssens PJ, Lavigne R, Mattheus W, Chibeu A, Hertveldt $\mathrm{K}$, Mast J, et al. Genomic analysis of Pseudomonas aeruginosa phages LKD16 and LKA1: establishment of the phiKMV subgroup within the T7 supergroup. $J$ Bacteriol 2006; 188: 6924-6931, doi: 10.1128/JB.00831-06.

20. Ceyssens PJ, Mesyanzhinov V, Sykilinda N, Briers Y, Roucourt $B$, Lavigne $R$, et al. The genome and structural proteome of YuA, a new Pseudomonas aeruginosa phage resembling M6. J Bacteriol 2008; 190: 1429-1435, doi: 10.1128/JB.01441-07.

21. Garbe J, Wesche A, Bunk B, Kazmierczak M, Selezska K, Rohde C, et al. Characterization of JG024, a Pseudomonas aeruginosa PB1-like broad host range phage under simulated infection conditions. BMC Microbiol 2010; 10: 301, doi: 10.1186/1471-2180-10-301.

22. Ramsey DM, Wozniak DJ. Understanding the control of Pseudomonas aeruginosa alginate synthesis and the prospects for management of chronic infections in cystic fibrosis. Mol Microbiol 2005; 56: 309-322, doi: 10.1111/ j.1365-2958.2005.04552.x.

23. Goyal SM, Gerba CP, Bitton G. Phage ecology. New York: Wiley-Interscience Publication; 1987.

24. Wagner EM, Hewlett M. Basic virology. Blackwell Science Inc.; 1999.

25. Watanabe R, Matsumoto T, Sano G, Ishii Y, Tateda K, Sumiyama $Y$, et al. Efficacy of bacteriophage therapy against gut-derived sepsis caused by Pseudomonas aeruginosa in mice. Antimicrob Agents Chemother 2007; 51: 446-452, doi: 10.1128/AAC.00635-06.

26. Hay ID, Remminghorst $U$, Rehm BH. MucR, a novel membrane-associated regulator of alginate biosynthesis in Pseudomonas aeruginosa. Appl Environ Microbiol 2009; 75: 1110-1120, doi: 10.1128/AEM.02416-08.

27. Silva JL, Hirata RDC, Hirata MH. Bacteriophage: laboratorial diagnosis and phage therapy. Braz J Microbiol 2009; 40: 547-549, doi: 10.1590/S1517-83822009000300017.

28. Deresinski S. Bacteriophage therapy: exploiting smaller fleas. Clin Infect Dis 2009; 48: 1096-1101, doi: 10.1086/ 597405. 